Original Research Paper

\title{
Neurophysiological Mechanisms of Rapidity Quality Development in Female Students Going in for Basketball and Volleyball at Sports Clubs
}

\author{
Angelina Shakhanova, Aida Cheuzheva, Ekaterina Kalnaya and Tatiana Petrova \\ Adyghe State University (AGU), Maikop, Russian Federation
}

Article history

Received: 21-10-2015

Revised: $13-05-2016$

Accepted: 14-05-2016

Corresponding Author: Angelina Shakhanova

Adyghe State University

(AGU), Maikop, Russian

Federation

Email: Nisadgu@yandex.ru

\begin{abstract}
Using the "NS-Pshykho Test" certified software package (Ivanovo, Russia), we studied the measurements of Simple Hand-Eye Response time (SHER), tapping test frequency, time of Reaction To Moving Object (RMO), Critical Flicker Fusion Frequency (CFFF) in female students going and not going in for sports (volleyball, basketball) at sports clubs. The basis of neuromotor state is formed by variants of classical techniques of chronoreflexometry characterizing the overall tone, mobility, lability, strength of CNS (SHER, tapping test), balance of activation-inhibitory processes (RMO), Rapidity and Visual Perception Clarity (CFFF). Strategic processing of results was made by means of Statistica 6.0 software and Microsoft Excel's built-in statistical function. In the process of training macro-cycles a higher level of reserves and rapidity quality develops and correction sensory level increases in female students going in for sports, especially when practicing basketball. However volleyball classes are more conducive to optimizing the balance of activation-inhibitory processes, which improves the possibility to regulate voluntarily manifestations of sensory and motor functions and enables to anticipate more effectively possible trajectory of the ball movement and speed up the response training. The independent sources present primarily fragmentary data on psychophysiological and psycho-emotional features of highly-qualified sportsmen. Several studies examine the properties of the Central Nervous System (CNS) as a limiting factor of sports activity that determines the functional and psychological preparedness of sportsmen, their model of motor abilities, especially in situational sports. There are no analogues of studying neuromotor characteristics of rapidity quality development in female students playing sports at specialized sports clubs and focused on maintaining a healthy lifestyle rather than achieving high sports results. The obtained results differ from the results previously reported by us for highly qualified sportsmen. Higher qualitative and quantitative characteristics of the functional state of the central nervous system demonstrated by qualified athletes as a result of long-term morpho-functional specialization of the body in comparison to sportsmen, who did not have sports category, prove their prognostic significance in achieving a high level of physical performance. Used modern tools of neuromotor diagnosis can be effectively used in monitoring the CNS functional state during sports training and timely correction of the training process.
\end{abstract}

Keywords: Central Nervous System, Adaptation, Neuromotor Status, Neuromotor Characteristics of Rapidity Quality, Female Students, Basketball, Volleyball

\section{Introduction}

The preservation and strengthening of students' health currently turns into a significant factor of socio- cultural and economic policy of any state. Sport as a part of a healthy lifestyle among students is becoming an important component that characterizes the development of society in modern conditions. The rate of perception 
and processing of incoming information and the speed of motor actions depend on the qualitative and quantitative characteristics of the functional reserves of the speed (Calmet et al., 2010; Seth, 2014).

An athlete must be able quickly to perceive and process information, which plays a significant role in obtaining positive results (Maly et al., 2011; Braulio et al., 2013). This is especially important for team sports that dominate among students. Training and competitive activities within team sports require intensive development of general and speed endurance, reaction rapidity, due to huge amount of information processed fast under the time pressure in a continuously changing situation (Dicks et al., 2011; Drid et al., 2012).

The individual characteristics within physiological processes in the nervous and muscular systems are the base for the rapidity quality manifestations. At the same time the central nervous system is the main element of the hierarchical structure of functional systems providing the adaptation of the body (Merchant et al., 2013; Perez et al., 2013). The problem of influence made by physical activities on the functional state within the nervous system is one of the central problems in studying patterns and adaptation of the body to the effects of physical and sport activity (Dicks et al., 2011; Friston, 2012). The level of lability and mobility of nervous processes, rate of muscle component reaction determine physiological reserves and rapidity development quality. Developed sensorimotor ability provides for operational thinking, capability for correct decision-making and rapid mobilization of tactical combinations from memory to address effectively tactical problems (Franchini et al., 2000; Bundzen et al., 2005; Drid et al., 2012). Some researchers consider properties of the nervous system as a limiting factor of sports activities, defining the functional and psychological preparedness of athletes, the model of their motor abilities, especially in situational sports (Dicks et al., 2011). Depending on the CNS functional state, adaptation to training a competitive activity has significant differences in efficacy and physiological values (Merchant et al., 2013). The features of neurophysiological status of persons engaged in physical culture and sports should be taken into account by the coach when determining the amount and intensity of workouts (Bundzen et al., 2005; Drid et al., 2012).

The reviewed literature contains insufficient information about the role of the functional state of the nervous system in the formation of a functional adaptive system to physical loads in game sports in students. As a rule, this information concerns qualified athletes, mainly male students. It actualizes the study of the neurophysiological status of female students not going in for sports and playing basketball and volleyball at sports clubs in order to substantiate the role of sports activities in maintaining their mental and reproductive health. The need for a profound study of the problem resides in the fact that the individual neuromotor profile is practically ignored when staffing sports groups and determining the mode of training exercises.

Defining the neuromotor status can serve as a tool to determine the current functional status of the CNS. The data obtained can be used by a coach to optimize the training process in the prediction of overtraining and fatigue, to develop programs for targeted improvement of students' CNS functionality through sports training in order to maintain favorable dynamics of mental and physical performance.

\section{Research Methodology}

The indicators of the functional state of the nervous system were studied at the premises of the Physiology of Child Development laboratory of the Institute for Complex Problems of Adyghe State University (Russia) in female students of the faculties not related to sports, who regularly went in for sports at basketball and volleyball clubs. The use of longitudinal and differentiated approaches that are quite labor intensive, but highly representative is an important moment of the research methodology modification. In this research, 60 female students aged 17-24 years were engaged, including 20 basketball players (sports experience from 3 to 5 years); 20 volleyball players (sports experience from 2 to 7 years), 20 students with the traditional regulated motor mode $(2 \mathrm{~h}$ of physical training per week). They attended four basketball and volleyball training sessions per week. Each one lasted 1.5 to $3 \mathrm{~h}$. In basketball and volleyball sections most of the time (60 to $65 \%$ ) was assigned to the tactical and gaming activities; technical training took $25-30 \%$ of the time, with the time of general physical training making only $10-15 \%$.

All studies were carried out with the written consent of the tested persons. In order to standardize the conditions, the study was conducted at a temperature of comfort $\left(18-20^{\circ} \mathrm{C}\right)$ before noon $(9: 00-12: 00 \mathrm{AM})$.

The study of peculiarities of neuromotor characteristics was carried out using the "NS-Pshyco Test" computer system of the "Neurosoft" Company, Ivanovo, Russia. The system included an advanced psycho physiological tester and an IBM-compatible personal computer. They investigated the rates of Response to a Moving Object (RMO), Critical Frequency of Flicker Fusion (CFFF), Simple Hand-Eye Response (SHER) and the tapping test, which are the variants of classical techniques of chronoreflexometry. They characterize the overall tone, lability, mobility, strength of CNS, which is also considered one of the most common indicators for testing the rapidity quality.

To assess SHER, we showed the tested person red signals, with the appearance of which, he was to press a button quickly. The minimum and maximum time interval 
between two successive presentations of the signal ranged from one to three seconds. The timeout signal was $5 \mathrm{sec}$.

To assess the balance of nervous processes in students, the rate of Response to a Moving Object (RMO) was used. In order to determine RMO, the tested person was shown a white circle on the screen, which had two markers: Red and green. Starting from the red marker, red filling was spreading at a speed of 15 $\mathrm{mm} / \mathrm{sec}$. At the time the border passed the green marker, the tested person was to press the special button quickly. The signal was displayed on the computer screen 10 times. The examination report included the received test data and results of their processing in the form of the absolute average time of RMO and the accuracy rate of motor responses. The absolute and relative (\%) number of precise, advanced and retarded reactions were indicated in the results and a conclusion on the predominance of excitatory and inhibitory processes was made.

Critical Frequency of Flicker Fusion (CFFF) reflects the strength and lability of the nervous system depending on the rapidity and clarity of visual perception. The CFFF assessment technique is a psycho-physiological method, which consists in sequential presentation of the discrete light stimuli of decreasing frequency to examinee, when it is required to determine the frequency of light flashes, at which they merge and are subjectively perceived as continuous even illumination. The minimum frequency of flashes per second, at which a flicker fusion occurs, is called critical flicker fusion frequency. A range of scales aimed at determining the individual level of the nervous system lability is presented: Low lability-less than $36.5 \mathrm{~Hz}$; medium lability $-36.6-43.3 \mathrm{~Hz}$; high lability $-43.4 \mathrm{~Hz}$ and above.

One of the integral indicators of lability, mobility, the CNS strength and the response rate of the neuromotor apparatus is the tapping test, based on the measurement of the hand movement rate dynamics. Tapping test is used for the diagnosis of strength and functional mobility of nervous processes by measuring the dynamics of the hand movements' rate. To perform a tapping test, it was necessary to tap the plate with a contact pointer as often as possible until the end of the test. The total test time was $60 \mathrm{sec}$. According to the obtained figures, a curve was drawn, characterizing the overall health of the tested person, the strength of the nervous system and the rapidity quality.

\section{Results and Discussion}

Game sports imply stepped-up requirements for speed and accuracy parameters of the movements performed on the playing field. The most important indicator describing the rate of the excitation and inhibition processes in the central nervous system, the ability to ensure high accuracy of the work performed is the SHER time.
In sport physiology, the reaction time is used as an indicator of the physiological condition of the central nervous system characterizing the rapidity quality development, increase in the sensory correction level in the game training conditions. It is shown that in the process of training sessions, the response time is overwhelmingly reduced (in $71.3 \%$ of the survey sample), as well as its variability. On the contrary, sport hyperkinesia extends the response time and reduces its resistance (in $28.7 \%$ of the survey sample).

The theoretical analysis presented in the works by (Friston, 2012; Merchant et al., 2013) shows that even with the most careful observance of all the methodological requirements and the highest level of fitness, the time of the simple sensorimotor response of a person to a pre-agreed signal cannot be below a certain physiological limit or "irreducible minimum". It consists of the time required for the formation of the corresponding generating capacity in receptors, transferring it by afferent pathways, processing in the CNS structures, carrying the signal to the executive organs and exercising the movement.

The comparative analysis of the time of a simple hand-eye response showed that the lowest SHER values were observed in basketball players $-181.11 \pm 0.5 \mathrm{~Hz}$ compared to $202.44 \pm 0.4 \mathrm{~Hz}$ in volleyball-players $(p<0.05)$ and $228.4 \pm 1.4 \mathrm{~Hz}$ in the students not engaged in sports activities $(\mathrm{p}<0.05)$. Reduction of simple sensory-motor response time in the female students going in for sports indicates the high development of the rapidity quality and the increase in of the sensor correction level during sports game training compared to female students not engaged in sports activities. This is indicative of a significant improvement in the current functional state of the CNS in the female students playing sports. Presumably, higher speed of response is associated with the shortening time of the "central delay" because it largely reduces the latency time of the reaction as a result of training for rapidity, as the time is extremely short to transfer excitation from receptors to nerve centers and then to muscles.

The comparative analysis of SHER results shows that in the circumstances of basketball training, the most favorable conditions are created for the high quality of rapidity development. This is due to the specific training activity in basketball, requiring agility of motor programs, a high level of concentration, high sensitivity of the motor analyzer in the wrist joint. Herewith, movements in volleyball are less intense than in basketball (Joy et al., 2006).

The maximum frequency of movement scan beone of the integral indicators of rapidity. A number of papers state that increasing the hands 'rate-related capability with special training is associated with a package of central and peripheral alterations, morpho-physiological alterations, in particular with the increasing number of fast-twitch 
muscle fibers, the improving coordination between synergist and antagonist muscles, etc. (Seth, 2014).

The maximum rate of movements $(8.2 \pm 0.6 \mathrm{~Hz})$, indicating a strong type of the nervous system, was shown by basketball players $(63.5 \%$ versus $38.6 \%$ of volleyball female players and $19.8 \%$ of female students not engaged in sports activities). The tapping frequency registered among the volleyball players was $7.4 \pm 0.3 \mathrm{~Hz}$, which indicates the prevalence of people with a medium type of the nervous system in this group $(49.4 \%$ versus $28.7 \%$ of female basketball players and $32.4 \%$ of female students not engaged in sports activities). This allows stating that the bodies of female basketball players are able to withstand more intense and prolonged stress, than the bodies of volleyball players and, particularly, female students not engaged in sports activities. This can be explained by the fact that basketball requires the highest possible development of speed and rate of movements in the conditions of time deficiency.

Female students not engaged in sports activities had the frequency of tapping $4.9 \pm 1.3 \mathrm{~Hz}$, which indicates the prevalence of the weak type of the nervous system (47.8 Vs. $7.8 \%$ in female basketball players and $12 \%$ in female volleyball players). Against this background, the phases of fatigue and strain of the body in the process of physical and educational activities develop faster in female students not engaged in sports activities. Perhaps, individuals with a strong nervous system have a "reserve" capacity for mobilization. Having started an exercise at almost the same rate, as the person with an average strength of the nervous system, those with a strong nervous system can increase the rate due to willful effort, which cannot be done by the others.

The CFFF indicator is based on the eye's ability to perceive low-frequency periodic interruptions of the light stimulus. CFFF measured by the number of light flashes per second varies in a human from 14 to $70 \mathrm{~Hz}$, reflecting the individual characteristics of the brain nervous processes, present functional state of the central nervous system, rapidity, visual perception clarity. A CFFF test is the most accurate technique to diagnose the state of fatigue out of all the methods associated with predominantly mental and physical labor, activities with a large volume of small and precise movements, requiring eyestrain.

The values of this indicator are closely associated with the lability and strength of the nervous system. The highest values in the CFFF study were identified in female basketball players $-44.3 \pm 9.84 \mathrm{~Hz}$, which indicates the prevalence of individuals with high lability and strength of the nervous system. The female volleyball playershadan average level of strength and lability of the nervous system according to the CFFF test results $39.34 \pm 1.89 \mathrm{~Hz}(\mathrm{p}<0.05)$. In the course of regular training in basketball higher level of CFFF contributes to better orientation at the basketball court, provides continuous tracking of the ball and the increased accurate throws of the ball into the hoop of basketball players. In volleyball players the increase in CFFF values results in the enhanced number of more accurate spikes and bends.

The CFFF index in female students not engaged in sports activities was equal to $34.83 \pm 0.92 \mathrm{~Hz}(\mathrm{p}<0.05)$, which indicates a low level of strength and lability of the nervous system, lower level of rapidity and visual perception clarity in comparison with their peers, going in for sports. Higher CFFF values in female volleyball and particularly basketball players, in comparison to the students not engaged in sports activities, indicate their higher speed and visual perception clarity, high ability of differentiating response and high sensitivity of the visual analyzer to the effects of perceptual stimuli.

The most important indicator of CNS functional state is the RMO indicator, which reflects the growth of mobility, balance and concentration, both in space and in time, of excitatory and inhibitory processes (Anderson, 1997; Yoon, 2002). The development of an optimal rate of Response to a Moving Object (RMO) in sport players enables to anticipate the likely movement path of the ball, teammates and opponents on the playing field and accelerates the preparation of response. The practice shows that athletes who have an optimal balance in activation-inhibitory processes act effectively on the playing field and have less emotional stress. This is especially important for basketball, as players are constantly in the situation of rapid response to changing conditions at the playing ground, the complexity of which is determined by the multiplicity of alternatives and rigid time limits for making a decision.

The comparative analysis showed that the highest values of precise response were observed in female students playing volleyball $-53.8 \%$, compared with female students playing basketball $(47.95 \%)$ and female students not engaged in sports activities $(25.4 \%)$. The advancing strategy was used by $25.0 \%$ of the surveyed female volleyball players, compared to $28.5 \%$ of female basketball players and $27.2 \%$ of female students not engaged in sports activities. The highest values of the response lag were observed in students not engaged in sports activities (47.4\%), while $23.9 \%$ of female basketball players and $21.3 \%$ of female volleyball players used the strategy of delay. The presence of the contingent with advancing reactions indicates the predominance of the excitatory process. Delayed response indicates gradual inclusion of the body in the work on against the background of prevalence of the braking process. The contingent with a high percentage of accurate responses, particularly in female volleyball players, points to the optimization of the balance of activation-inhibitory processes in game sports, which is a prerequisite for the success of the game. 


\section{Conclusion}

The established differences in neurophysiological status' indicators of the surveyed female students going and not going in for sports reflect the direct dependence of the impact of the physical activity and its scope on the improvement of mechanisms of central movementcontrol link. Moreover, in terms of basketball training, the dominating contingent has the highly developed sensory correction, lability and functional mobility and strength of nervous processes. Whereas, in the study of Reactions to a Moving Object (RMO) and response strategies it has been found that playing volleyball is more conducive to optimizing the balance of activation-inhibitory processes, which is one of the determining factors in terms of high duration of optimum performance at the playing ground. Alongside with that, the presence in sports clubs of female students with low functional capacity of the nervous system (up to $28.7 \%$ ) may become an obstacle to the achievement of high sports results, if no measures are taken to develop strategies for differentiation of training loads, with regard to their age and individual characteristics. In general, the studies have shown that regular playing basketball and volleyball in female students who did not have any sports category results in extended functionality of the central nervous system, improved neuromotor apparatus, creation of favorable conditions for the rapidity quality development and more successful adaptation to physical and mental activity. Lower values of neuromotor performance status in female students, previously not involved in sports activities, point to the need to find mechanisms for improvement of the functional state of the central nervous system, aimed at optimizing the indicators of neuromotor status and strengthening the body's health.

\section{Acknowledgement}

The authors express their thanks to unknown referees for the careful reading and helpful comments.

\section{Funding Information}

The investigations have been carried out according to the plan of performing the research and development studies at the Adyghe State University.

\section{Author's Contributions}

All authors completed the paper together as well as read and approved the final manuscript.

\section{Ethics}

The authors have no conflicts of interest in the development of the research and publication of this article.

\section{References}

Anderson, M., 1997. Reaction time measures of speed of processing: Speed of response selection increases with age but speed of stimulus categorization does not. Brit. J. Dev. Psychol., 15: 145-157. DOI: 10.1111/j.2044-835X.1997.tb00731.X

Braulio, H.M., B.H. Branco, L.M. Massuça, L.V. Andreato and B.F. Marinho et al., 2013. Association between the rating perceived exertion, heart rate and blood lactate in successive judo fights (Randori). Asian J. Sports Med., 4: 125-130. PMID: 23802054

Bundzen, P.V., K.G. Korotkov, A.K. Korotkova, V.A. Mukhin and N.S. Priyatkin, 2005. Psychophysiological correlates of athletic success in athletes training for the olympics. Hum. Physiol., 31: 316-323. DOI: 10.1007/s10747-005-0051-y

Calmet, M., B. Miarka and E. Franchini, 2010. Modeling of grasps in judo contests. Int. J. Perform. Anal. Sport, 10: 229-240.

Dicks, M., L. Uehara and C. Lima, 2011. Deception, individual differences and penalty kicks: Implications for Goalkeeping in association football. J. Sports Sci. Coach, 6: 515-522. DOI: 10.1260/1747-9541.6.4.515

Drid, P., T. Trivić and S. Tabakov, 2012. Special judo fitness test-a review. Serb. J. Sports Sci., 6: 117-125.

Franchini, E., M.Y. Takito and M.A.P.D.M. Kiss, 2000. Somatotipo, composição corporal e força isométrica em diferentes períodos do treinamento em atletas de judôjuvenis, ("Somatotype, corporal composition and isometric strength on different periods of the training plan for junior judo athletes"). Revista Treinamento Desportivo Brasil, 5: 4-10.

Friston, K., 2012. Prediction, perception and agency. Int. J. Psychophysiol., 83: 248-252. PMID: 22178504

Joy, J., E. Geng, S. Eger, S. Ruff and Á. Kristjánsson et al., 2006. Attentional selection from competing stimuli in opposite visual fields: Effects on human visual cortex and control processes. J. Neurophysiol., 96: 2601-2612.

Maly, T., L. Malá, F. Zahálka, J. Baláš and M. Čada, 2011. Comparison of body composition between two elite women's volleyball teams. Acta Univ. Palacki Olomuc Gymn, 41: 15-22. DOI: 10.5507/ag.2011.002

Merchant, H., D. Harrington and W.H. Meck, 2013. Neural basis of the perception and estimation of time. Annu. Rev. Neurosci., 36 : 313-336. PMID: 23725000

Perez, O., R. Kass and H. Merchant, 2013. Trial time warping to discriminate stimulus-related from movement-related neural activity. J. Neurosci. Meth., 212: 203-210. PMID: 23147009 
Seth, A.K., 2014. A predictive processing theory of sensorimotor contingencies: Explaining the puzzle of perceptual presence and its absence in synesthesia. Cogn. Neurosci., 5: 97-118. PMID: 24446823
Yoon, J., 2002. Physiological profiles of elite senior wrestlers. Sports Med., 32: 225-233. PMID: 11929352 